\title{
The Boolean Rainbow Ramsey Number of Antichains, Boolean Posets and Chains
}

\author{
Hong-Bin Chen* \\ Department of Applied Mathematics \\ National Chung Hsing University \\ Taichung 40227, Taiwan \\ andanchen@gmail.com \\ Wei-Tian $\mathrm{Li}^{\ddagger}$ \\ Department of Applied Mathematics \\ National Chung Hsing University \\ Taichung 40227, Taiwan \\ weitianli@nchu.edu.tw
}

\author{
Yen-Jen Cheng ${ }^{\dagger}$ \\ Department of Mathematics \\ National Taiwan Normal University \\ Taipei 116325, Taiwan \\ yjc7755@gmail.com \\ Chia-An Liu \\ Department of Mathematics \\ Soochow University \\ Taipei 11102, Taiwan \\ liuchiaan8@gmail.com
}

Submitted: Sep 25, 2019; Accepted: Nov 2, 2020; Published: Nov 27, 2020

(C) The authors. Released under the CC BY-ND license (International 4.0).

\begin{abstract}
Motivated by the paper, Boolean lattices: Ramsey properties and embeddings Order, 34 (2) (2017), of Axenovich and Walzer, we study the Ramsey-type problems on the Boolean lattices. Given posets $P$ and $Q$, we look for the smallest Boolean lattice $\mathcal{B}_{N}$ such that any coloring of elements of $\mathcal{B}_{N}$ must contain a monochromatic $P$ or a rainbow $Q$ as an induced subposet. This number $N$ is called the Boolean rainbow Ramsey number of $P$ and $Q$ in the paper.

Particularly, we determine the exact values of the Boolean rainbow Ramsey number for $P$ and $Q$ being the antichains, the Boolean posets, or the chains. From these results, we also derive some general upper and lower bounds of the Boolean rainbow Ramsey number for general $P$ and $Q$ in terms of the poset parameters.
\end{abstract}

Mathematics Subject Classifications: 05C55, 05D05, 05D10

\section{Introduction}

A poset $P=\left(P, \leqslant_{P}\right)$ is a set $P$ equipped with a partial order $\leqslant_{P}$. For any two elements $a, b \in P$ if either $a \leqslant_{P} b$ or $b \leqslant_{P} a$ holds, then $a$ and $b$ are comparable, otherwise

\footnotetext{
*Supported by MOST 107-2115-M-035 -003 -MY2.

${ }^{\dagger}$ Supported by MOST 109-2811-M-003-524

${ }^{\ddagger}$ Supported by MOST-107-2115-M-005 -002 -MY2.
} 
they are incomparable. In this paper, we study the Ramsey-type problems of the wellknown poset, Boolean lattice, $\mathcal{B}_{n}$ whose underlying set is the collection of all subsets of $[n]:=\{1,2, \ldots, n\}([0]=\varnothing$ as a convention $)$ and the partial order is the inclusion relation on sets. The $k$-th level of $\mathcal{B}_{n}$ is the collection of all $k$-subsets of $[n]$, denoted by $\left(\begin{array}{c}{[n]} \\ k\end{array}\right)$. A family $\mathcal{F}$ of subsets is isomorphic to a poset $P$ if there exists an order-preserving bijection $\phi$ between $P$ and $\mathcal{F}$, i.e., $P \stackrel{\phi}{\longleftrightarrow} \mathcal{F}$ such that for any $x_{1}, x_{2} \in P, x_{1}<_{P} x_{2}$ if and only if $\phi\left(x_{1}\right) \subset \phi\left(x_{2}\right)$. If a family $\mathcal{F}$ contains a subfamily $\mathcal{G}$ isomorphic to $P$, we will say $\mathcal{F}$ contains $P$ as a strong (or induced) subposet $(\mathcal{F}$ contains $P$, for short), or say the subsets in $\mathcal{G}$ form a copy of $P$. A coloring ( $k$-coloring) of $\mathcal{B}_{n}$ is a mapping $c$ from $\mathcal{B}_{n}$ to a set of positive integers (to $[k]$ ). Given a coloring $c$ of $\mathcal{B}_{n}$, we say $\mathcal{B}_{n}$ contains a monochromatic $P$ under $c$ if there is a family of subsets of the same color containing $P$.

In the literature on Ramsey theory, the Ramsey problems have been greatly studied on the set systems (hypergraphs), the graphs, the planes, and the general posets. For example, see $[1,5,6,9,13,18]$. The following type of Ramsey problems on Boolean lattices has been recently studied by Axenovich and Walzer [2]:

Question 1. Given posets $P_{1}, \ldots, P_{k}$, can one find the least integer $n$ such that for any $k$-coloring of $\mathcal{B}_{n}$, it always contains a monochromatic $P_{i}$ of color $i$ for some $i$ ?

In their paper, they called such a number the poset Ramsey number. Since there are many Ramsey properties investigated on the families of posets with various parameters, to make it more precise, we suggest the name the Boolean Ramsey number and use it in this paper. Let us define the term formally below:

Definition 2. Given posets $P_{1}, \ldots, P_{k}$, the Boolean Ramsey number $R\left(P_{1}, \ldots, P_{k}\right)$ is the minimum integer $n$ such that for any $k$-coloring of $\mathcal{B}_{n}$, it always contains a monochromatic $P_{i}$ of color $i$ for some $i$. Moreover, if $P_{i}=P$ for all $1 \leqslant i \leqslant k$, then we use $R_{k}(P)$ to denote $R\left(P_{1}, \ldots, P_{k}\right)$.

Using a theorem in [16], Axenovich and Walzer proved that the number $R_{k}(P)$ is finite in $[2]$ :

Theorem 3. (Theorem 6, [2]) For any poset that is not an antichain, $R_{k}(P)=\Theta(k)$.

Including the above theorem, Axenvoich and Walzer obtained a series of results on the Boolean Ramsey number for the target posets being the Boolean lattices in [2], which are improved by $\mathrm{Lu}$ and Thompson [15] very recently.

Given a coloring $c$ of $\mathcal{B}_{n}$, we say $\mathcal{B}_{n}$ contains a rainbow $P$ if it contains $P$ and all the subsets forming $P$ are of distinct colors under $c$. Imitating the rainbow Ramsey number in graph theory, we give the following definition:

Definition 4. Given two posets $P$ and $Q$, the Boolean rainbow Ramsey number $R R(P, Q)$ is the minimum integer $n$ such that for any coloring $c$ of $\mathcal{B}_{n}$, it always contains either a monochromatic $P$ or a rainbow $Q$ as a subposet. 
A result by Johnston, Lu, and Milans [12] implies that $R R(P, Q)$ is finite for any $P$ and $Q$. They proved that for $n$ sufficiently large, any coloring of $\mathcal{B}_{n}$ contains either a monochromatic Boolean algebra $B_{a l g(r)}$ or a rainbow $B_{a l g(s)}$ for any given positive integers $r$ and s. A Boolean algebra $B_{a l g(r)}$ in $\mathcal{B}_{n}$ is such a family $\left\{S_{0} \cup\left(\cup_{i \in I} S_{i}\right) \mid I \subset[r]\right\}$, where $S_{0}, S_{1}, \ldots, S_{r}$ are pairwise disjoint subsets of $[n]$ with $S_{i} \neq \varnothing$ for $i \geqslant 1$.

Theorem 5. (Theorem 5, [12]) For $N \geqslant r 2^{(2 r+1) 2^{s-1}-2}, \mathcal{B}_{N}$ contains either a rainbow $B_{\text {alg(r) }}$ or a monochromatic $B_{\text {alg(s) }}$ under any coloring.

Containing the Boolean algebra $B_{a l g(r)}$ implies containing $\mathcal{B}_{r}$ since $B_{a l g(r)}$ is isomorphic to $\mathcal{B}_{r}$. So $R R(P, Q)$ is finite when $P$ and $Q$ are both Boolean lattices. In addition, Trotter [17] introduced the 2-dimension of a poset $P, \operatorname{dim}_{2}(P)$, the minimum number $n$ for which $\mathcal{B}_{n}$ contains $P$ and proved that $\operatorname{dim}_{2}(P)$ is finite for any poset $P$. As a consequence, $R R(P, Q)$ exists for any $P$ and $Q$.

Analogous to [2], Cox and Stolee [7] studied the existence of the monochromatic weak subposets in the Boolean lattices. Here a weak subposet means an injection from the poset to the Boolean lattice, which preserves the inclusion relation but not necessary the non-inclusion relation. A very recent paper [4] by the third author and others presents the results on the Ramsey properties of both types of subposets in the Boolean lattices.

In this paper, we study the strong version of the Boolean rainbow Ramsey number and the relations between it and the Boolean Ramsey number. We determine the exact values of $R R(P, Q)$ for specific posets and use the results to derive the upper and lower bounds for $R R(P, Q)$ for general $P$ and $Q$. We focus on the antichains $A_{n}$, the Boolean posets $B_{n}$, and the chains $C_{n}$, where $A_{n}$ is a set of $n$ pairwise incomparable elements, $B_{n}$ is isomorphic to $\mathcal{B}_{n}$, and $C_{n}$ is a set of $n$ mutually comparable elements. In the paper, the scribe $\mathcal{B}_{n}$ refers to the underlying Boolean lattice we color, and the capital $B_{n}$ refers to the desired monochromatic or rainbow Boolean posets. Table 1 is the summary of our results on $R R(P, Q)$ when $P$ and $Q$ are one of the three types of posets.

\begin{tabular}{|c|c|c|}
\hline$P$ & $Q$ & $R R(P, Q)$ \\
\hline$A_{m}$ & $A_{n}$ & $\left.\min \left\{N \mid \begin{array}{c|}N \\
\lfloor N / 2\rfloor\end{array}\right) \geqslant(m-1)(n-1)+1\right\}$. \\
\hline$A_{2}$ & $C_{n}$ & $n$ \\
\hline$B_{m}$ & $B_{1}$ & $m$ \\
\hline$B_{1}$ & $B_{n}$ & $2^{n}-1$ \\
\hline$C_{m}$ & $A_{n}$ & $\left\{\begin{array}{c}n+2, \\
(m-1)(n-1)+2,\end{array} \quad \begin{array}{l}m=n=2, \text { or } m \geqslant 3 \\
(m-1)\left(2^{n}-1\right)\end{array}\right.$ \\
\hline$C_{m}$ & $B_{n}$ & $(m-1)(n-1)$ \\
\hline$C_{m}$ & $C_{n}$ & $(m \geqslant 2$ \\
\hline
\end{tabular}

Table 1: $R R(P, Q)$ of antichains, Boolean posets, and chains

The organization of the remaining sections in the paper is the following. In Section 2, we first determine the values of the Boolean rainbow Ramsey numbers for antichains or 
chains, and deduce some lower bounds from those results. In Section 3, we give an upper bound on $R R\left(B_{m}, B_{n}\right)$ for general $m$ and $n$, and determine the values of $R R\left(B_{1}, B_{n}\right)$ and $R R\left(B_{m}, B_{1}\right)$ as listed in Table 1 . Using the idea of determining $R R\left(B_{1}, B_{n}\right)$, we manage to solve $R R\left(C_{m}, B_{n}\right)$, which is also demonstrated in this section. The last section contains discussions for the relations of $R_{k}(P), R R(P, Q)$, and the forbidden subposet problems.

\section{Chains, antichains, and the general lower bounds}

When $P$ and $Q$ are both chains or both antichains, the Boolean rainbow Ramsey numbers can be determined by simple arguments.

Proposition 6. For the chains, we have $R R\left(C_{m}, C_{n}\right)=(m-1)(n-1)$.

Proof. For $N<(m-1)(n-1)$, we give a coloring $c$ to $\mathcal{B}_{N}$ by coloring the subsets with $i$ in the consecutive $m-1$ levels $\left(\begin{array}{c}{[N]} \\ (i-1)(m-1)\end{array}\right) \cup\left(\begin{array}{c}{[N]} \\ (i-1)(m-1)+1\end{array}\right) \cdots \cup\left(\begin{array}{c}{[N]} \\ i(m-1)-1\end{array}\right)$ for $1 \leqslant i \leqslant\left\lceil\frac{N}{m-1}\right\rceil$. Since a chain $C_{m}$ in the Boolean lattices consists of $m$ subsets of distinct sizes, $\mathcal{B}_{N}$ does not contain a monochromatic $C_{m}$ under $c$. In the coloring $c$, the number of color classes is at most $n-1$, so $\mathcal{B}_{N}$ does not contain a rainbow $C_{n}$ as well. When $N \geqslant(m-1)(n-1)$, consider any coloring of the chain $\varnothing \subset[1] \subset[2] \subset \cdots \subset[N]$ in $\mathcal{B}_{N}$. By the pigeonhole principle, it contains at least $n$ subsets of distinct colors or at least $m$ subsets of the same color, as desired.

Let $N_{m, n}$ be the minimum integer such that $\left(\begin{array}{c}N_{m, n} \\ \left\lfloor N_{m, n} / 2\right\rfloor\end{array}\right) \geqslant(m-1)(n-1)+1$.

Proposition 7. For the antichains, we have $R R\left(A_{m}, A_{n}\right)=N_{m, n}$.

Proof. For $N<N_{m, n}$, partition $\mathcal{B}_{N}$ into $\left(\begin{array}{c}N \\ \lfloor N / 2\rfloor\end{array}\right)$ chains, $\mathcal{C}_{1}, \ldots, \mathcal{C}_{\left(\begin{array}{c}N \\ \lfloor N / 2\rfloor\end{array}\right)}$ using the symmetric chain decompositions $[3,10]$. Then color every $m-1$ chains $\mathcal{C}_{(i-1)(m-1)+1}, \ldots, \mathcal{C}_{i(m-1)}$ with $i$. Hence any antichain in a color class has size at most $m-1$. Also, the coloring contains at most $n-1$ colors. Thus, there is no rainbow $A_{n}$. For $N \geqslant N_{m, n}$, the level $\left({ }_{\lfloor N / 2\rfloor}^{[N]}\right)$ contains at least $(m-1)(n-1)+1$ subsets. Then any coloring of $\mathcal{B}_{N}$ contains at least $m$ subsets of the same color or $n$ subsets of distinct colors in the level $\left(\left[\begin{array}{c}{[N]} \\ {[N / 2\rfloor}\end{array}\right)\right.$.

The height and width of a poset $P, h(P)$ and $w(P)$, are the sizes of a maximum chain and antichain of $P$, respectively. It is clear $R R(P, Q) \geqslant R R\left(P^{\prime}, Q^{\prime}\right)$ if $P^{\prime}$ and $Q^{\prime}$ are subposets of $P$ and $Q$, respectively. The following bounds on $R R(P, Q)$ for general $P$ and $Q$ are the consequences of Proposition 6 and Proposition 7.

Corollary 8. For any posets $P$ and $Q$, we have $R R(P, Q) \geqslant(h(P)-1)(h(Q)-1)$ and $R R(P, Q) \geqslant N_{w(P), w(Q)}$.

For $P$ and $Q$ being different types of posets, the problem becomes much harder and more interesting. we manage to determine $R R\left(C_{m}, A_{n}\right)$ in Theorem 9 and give an upper bound for $R R\left(A_{m}, C_{n}\right)$ in Theorem 11 . 
Theorem 9. For the chains and antichains, we have

$$
R R\left(C_{m}, A_{n}\right)= \begin{cases}n+2, & m=2 \text { and } n \geqslant 3 \\ (m-1)(n-1)+2, & m=n=2, \text { or } m \geqslant 3 \text { and } n \geqslant 2 .\end{cases}
$$

To prove Theorem 9, we need more preparations. Two families $\mathcal{F}$ and $\mathcal{G}$ of subsets are said to be incomparable if for any $F \in \mathcal{F}$ and $G \in \mathcal{G}$, neither $F \subseteq G$ nor $G \subseteq F$. The following structure in the Boolean lattices will help us to determine $R R\left(C_{m}, A_{n}\right)$.

Lemma 10. Let $m \geqslant 2$ and $n \geqslant 2$ be integers. For $m \geqslant 3$, we can find $n$ pairwise incomparable chains $\mathcal{C}_{1}, \ldots, \mathcal{C}_{n}$ with $\left|\mathcal{C}_{i}\right|=(m-1)(i-1)+1$ in $\mathcal{B}_{(m-1)(n-1)+2}$. When $m=2$, this also holds for $n=2$. For $m=2$ and $n \geqslant 3$, we can find such a collection of chains in $\mathcal{B}_{n+2}$.

Proof. We prove the theorem by induction on $n$ and constructing the families of chains recursively. Fix $m \geqslant 3$. For $n=2$, we take the two chains $\mathcal{C}_{1}=\{\{m\}\}$ and $\mathcal{C}=$ $\{\{m+1\} \cup[j] \mid 0 \leqslant j \leqslant m-1\}$ contained in $\mathcal{B}_{m+1}$. Suppose we already have pairwise incomparable chains $\mathcal{C}_{1}, \ldots, \mathcal{C}_{n}$ in $\mathcal{B}_{(m-1)(n-1)+2}$ for some $n \geqslant 2$. In $\mathcal{B}_{(m-1) n+2}$, take $\mathcal{C}_{i}^{\prime}=$ $\left\{[(m-1) n+1]-X \mid X \in \mathcal{C}_{i}\right\}$ for $1 \leqslant i \leqslant n$ and $\mathcal{C}_{n+1}^{\prime}=\{\{(m-1) n+2\} \cup[j] \mid 0 \leqslant j \leqslant$ $(m-1) n\}$. If $\mathcal{C}_{i}^{\prime}$ and $\mathcal{C}_{j}^{\prime}$ are not incomparable for some $i<j \leqslant n$, say $Y_{1}=[(m-1) n+1]-X_{1}$ in $\mathcal{C}_{i}^{\prime}$ is a subset of $Y_{2}=[(m-1) n+1]-X_{2}$ in $\mathcal{C}_{j}^{\prime}$, then $X_{2}$ in $\mathcal{C}_{j}$ is a subset of $X_{1}$ in $\mathcal{C}_{i}$. This contradicts the incomparability of $\mathcal{C}_{i}$ and $\mathcal{C}_{j}$. Note that $(m-1) n+1>(m-1)(n-1)+2$ if $m \geqslant 3$. So each set in $\mathcal{C}_{i}^{\prime}$ for $1 \leqslant i \leqslant n$ contains $(m-1) n+1$ which is not in any set in $\mathcal{C}_{n+1}^{\prime}$. Also, $(m-1) n+2$ is in every set in $\mathcal{C}_{n+1}^{\prime}$ but not in any set in $\mathcal{C}_{i}^{\prime}$ for $1 \leqslant i \leqslant n$. Therefore, $\mathcal{C}_{1}^{\prime}, \ldots, \mathcal{C}_{n+1}^{\prime}$ are incomparable. For $m=n=2$, the above construction consists of $\{\{2\}\}$ and $\{\{3\},\{1,3\}\}$, which are incomparable.

Now for $m=2$ and $n \geqslant 3$, we first take $\mathcal{C}_{1}=\{\{1,2\}\}$ and $\mathcal{C}_{2}=\{\{4\},\{1,4\}\}$ as the desired incomparable chains in $\mathcal{B}_{4}$. Suppose we already have pairwise incomparable chains $\mathcal{C}_{1}, \ldots, \mathcal{C}_{n}$ in $\mathcal{B}_{n+2}$ for some $n \geqslant 3$. In $\mathcal{B}_{n+3}$, take $\mathcal{C}_{i}^{\prime}=\left\{[n+2]-X \mid X \in \mathcal{C}_{i}\right\}$, for $1 \leqslant i \leqslant n$, and $\mathcal{C}_{n+1}^{\prime}=\{\{n+3\} \cup[j] \mid 0 \leqslant j \leqslant n\}$. Similar to the case of $m \geqslant 3, \mathcal{C}_{1}^{\prime}, \ldots, \mathcal{C}_{n}^{\prime}$ are pairwise incomparable. The argument of incomparability of $\mathcal{C}_{n^{\prime}+1}$ and $\mathcal{C}_{i}^{\prime}, i \leqslant n$ is slightly different from the case of $m \geqslant 3$ : we have $n+2$ in each set in $\mathcal{C}_{i}^{\prime}$ for $1 \leqslant i \leqslant n-1$ but not in any set in $\mathcal{C}_{n+1}^{\prime}, n+1$ in each set in $\mathcal{C}_{n}^{\prime}$ but not in any set in $\mathcal{C}_{n+1}^{\prime}$, and $n+3$ in each set in $\mathcal{C}_{n+1}^{\prime}$ but not in any set in $\mathcal{C}_{i}^{\prime}$ for $1 \leqslant i \leqslant n$. Hence $\mathcal{C}_{1}^{\prime} \ldots, \mathcal{C}_{n+1}^{\prime}$ are pairwise incomparable.

Proof of Theorem 9. We use the constructions in Lemma 10 to show $R R\left(C_{2}, A_{n}\right) \leqslant n+2$ for $n \geqslant 3$, and $R R\left(C_{m}, A_{n}\right) \leqslant(m-1)(n-1)+2$ for $m=n=2$, or for $m \geqslant 3$.

Given $m, n \geqslant 2$, we consider an arbitrary coloring on $\mathcal{B}_{N}$ with $N=(m-1)(n-1)+2$ if $m \geqslant 3$ or $m=n=2$, or with $N=n+2$ if $m=2$ and $n \geqslant 3$. We have a family of pairwise incomparable chains $\mathcal{C}_{1}, \ldots, \mathcal{C}_{n}$ in $\mathcal{B}_{n}$ as described in Lemma 10. In both cases of $N$, for $i \geqslant 2$, there exist at least $i$ colors on the subsets in $\mathcal{C}_{i}$, otherwise it contains a monochromatic $C_{m}$. However, if there are $i$ colors on the subsets in $\mathcal{C}_{i}$, then we can pick a set from each chain $\mathcal{C}_{1}, \mathcal{C}_{2}, \ldots, \mathcal{C}_{n}$ in order so that all sets are of distinct colors. The $n$ sets form a rainbow $A_{n}$ since all the chains are incomparable. 
Let us establish the lower bound $R R\left(C_{2}, A_{n}\right)>n+1$ for $n \geqslant 3$. For $0 \leqslant i \leqslant n+1$, we color every subset in $\left(\begin{array}{c}{[n+1]} \\ i\end{array}\right)$ with $i$. Clearly, there is no monochromatic $C_{2}$ under this coloring. If it contains rainbow $A_{n}$, then neither $\varnothing$ nor $[n+1]$ can be in the rainbow antichain. So the rainbow $A_{n}$ must contain a set $X_{i} \in\left(\begin{array}{c}{[n+1]} \\ i\end{array}\right)$ for $1 \leqslant i \leqslant n$. However, if $X_{1} \not \subset X_{n}$, then $X_{n}=[n+1]-X_{1}$ and any other $X_{i}$ either contains $X_{1}$ or is contained in $X_{n}$. As a consequence, the rainbow $A_{n}$ does not exist.

Next, we show $R R\left(C_{m}, A_{n}\right)>(m-1)(n-1)+1$ for $m=n=2$, or for $m \geqslant 3$ and $n \geqslant 2$. Let $N=(m-1)(n-1)+1$. For each $X \in \mathcal{B}_{N}$, color $X$ with $1+\left\lceil\frac{|X|}{m-1}\right\rceil$. This gives an $(n+1)$-coloring of $\mathcal{B}_{N}$ such that no color appears on $m$ subsets of distinct sizes. So, there is no monochromatic $C_{m}$ under the coloring. Meanwhile, colors 1 and $n+1$ appear only on $\varnothing$ and $[N]$, respectively. Any family containing subsets of $n$ distinct colors must contain at least one of the two sets, and thus cannot be an antichain $A_{n}$.

Recall $N_{m, n}$ is the minimum integer such that $\left(\begin{array}{c}N_{m, n} \\ \left\lfloor N_{m, n} / 2\right\rfloor\end{array}\right) \geqslant(m-1)(n-1)+1$.

Theorem 11. Given $m \geqslant 2$, we have

$$
R R\left(A_{m}, C_{n}\right) \leqslant(m-1)(n-2)+N_{m, 2}
$$

for all $n \geqslant 2$. Moreover, the equality holds for $n=2$ or $m=2$.

Proof. Fix $m \geqslant 2$. For $n=2$, to avoid a rainbow $C_{2}$ in the Boolean lattices $\mathcal{B}_{N}$ means that each subset has the same color as the empty set, and thus $\mathcal{B}_{N}$ is monochromatic. So, $\mathcal{B}_{N}$ contains a monochromatic $A_{m}$ for $N \geqslant N_{m, 2}$. On the other hand, for $N \leqslant$ $N_{m, 2}-1$, a monochromatic $\mathcal{B}_{N}$ contains neither monochromatic $A_{m}$ nor rainbow $C_{2}$. Hence $R R\left(A_{m}, C_{2}\right)=N_{m, 2}$. Note that this shows that the equality holds when $n=2$ as the claimed statement.

Claim: $R R\left(A_{m}, C_{n+1}\right) \leqslant R R\left(A_{m}, C_{n}\right)+m-1$ for any $n \geqslant 2$.

Suppose we already have $R R\left(A_{m}, C_{n}\right)=k$ for some $n \geqslant 2$. Consider any coloring $c$ of $\mathcal{B}_{k+m-1}$. Assume that there is no monochromatic $A_{m}$ under $c$. Then we show there is a rainbow $C_{n+1}$. Assume $c([k+m-1])=1$ and let $\mathcal{F}_{1}$ be the family of nonempty subsets of color 1 . Since the maximum size of an antichain in $\mathcal{F}_{1}$ is at most $m-1$, by the well-known Dilworth's theorem [8], we can partition $\mathcal{F}_{1}$ into at most $m-1$ disjoint chains, say $\mathcal{C}_{1}, \mathcal{C}_{2}, \ldots, \mathcal{C}_{\ell}$ with $\ell \leqslant m-1$. For each $\mathcal{C}_{i}$, let $x_{i}$ be a common element in each subset in $\mathcal{C}_{i}$. Let $S=[k+m-1] \backslash\left\{x_{1}, \ldots, x_{\ell}\right\}$. Observe that $|S| \geqslant k$ and the color of each subset of $S$, except for $\varnothing$, is not 1 . We define a new coloring $c^{\prime}$ of $\mathcal{B}_{S}$, the Boolean lattices formed by all subsets of $S$, by letting $c^{\prime}(X)=c(X)$ for all nonempty $X \subseteq S$ and $c^{\prime}(\varnothing)=c(S)$. Because $|S| \geqslant k$ and there is no monochromatic $A_{m}$ under $c^{\prime}$, we can find a rainbow chain $C_{n}$ in $\mathcal{B}_{S}$. Note that a rainbow chain cannot contain both $S$ and $\varnothing$. If this chain does not contain $\varnothing$, then it together with $[k+m-1]$ form a rainbow $C_{n+1}$ under $c$. Else the chain contains $\varnothing$, then replace $\varnothing$ with $S$. The new chain together with $[k+m-1]$ form a rainbow $C_{n+1}$ under $c$. Therefore $R R\left(A_{m}, C_{n+1}\right) \leqslant k+m-1=R R\left(A_{m}, C_{n}\right)+m-1$ as we claimed. Repeatedly using the last inequality $n-2$ times and the fact $R R\left(A_{m}, C_{2}\right)=N_{m, 2}$ we obtain the inequality in the theorem. 
Finally, let us show the equality for $m=2$. By simple calculation we have $(m-$ $1)(n-1)+N_{m, 2}=n$ when $m=2$. It suffices to show $R R\left(A_{2}, C_{n}\right) \geqslant n$. For $N<n$, we color the subsets $\varnothing$ and $[N]$ in $\mathcal{B}_{N}$ with the same color, and each of the remaining subsets with distinct colors. Clearly, there does not exist monochromatic $A_{2}$, and every maximal rainbow chain has size $N \leqslant n-1$. Therefore, the lower bound holds and the proof is completed.

Since the exact value of $R R\left(C_{m}, A_{n}\right)$ is known, we also have the following lower bound on $R R(P, Q)$ in terms of $h(P)$ and $w(Q)$.

Corollary 12. For any posets $P$ and $Q$, we have

$$
R R(P, Q) \geqslant \begin{cases}w(Q)+2, & h(P)=2 \text { and } w(Q) \geqslant 3 \\ (h(P)-1)(w(Q)-1)+2, & h(P)=w(Q)=2, \text { or } \\ & h(P) \geqslant 3 \text { and } w(Q) \geqslant 2\end{cases}
$$

\section{Results related to Boolean posets}

Recall that the 2-dimension of a poset, $\operatorname{dim}_{2}(P)$, is the minimum number $d$ such that the Boolean lattice $\mathcal{B}_{d}$ contains $P$ as a subposet. Thus, the following upper bound on $R R(P, Q)$ for general $P$ and $Q$ is immediate.

Fact 13. For any poset $P$ and $Q$, we have

$$
R R(P, Q) \leqslant R R\left(B_{\operatorname{dim}_{2}(P)}, B_{\operatorname{dim}_{2}(Q)}\right) .
$$

From the above inequality, it turns out that determining $R R\left(B_{m}, B_{n}\right)$ is a more fundamental task. In the following, we first give an upper bound on $R R\left(B_{m}, B_{n}\right)$ using the ordinary Boolean Ramsey numbers $R_{k}\left(B_{m}\right)$, and then present the exact value of $R R\left(B_{m}, B_{n}\right)$ for $m=1$ or $n=1$.

Theorem 14. For the Boolean posets $B_{m}$ and $B_{n}$, we have

$$
R R\left(B_{m}, B_{n}\right) \leqslant \sum_{k=1}^{2^{n}-1} R_{k}\left(B_{m}\right)
$$

Proof. Let $N=\sum_{k=1}^{2^{n}-1} R_{k}\left(B_{m}\right)$. Consider a coloring $c$ on $\mathcal{B}_{N}$. We assume that $\mathcal{B}_{N}$ does not contain a monochromatic $B_{m}$ under $c$, and then prove there exists a rainbow $B_{n}$.

Let us arrange the nonempty subsets of $[n]$ in the order $I_{1}, \ldots, I_{2^{n}-1}$ such that $\left|I_{k}\right| \leqslant$ $\left|I_{k^{\prime}}\right|$ if $k<k^{\prime}$. Partition $[N]$ into $2^{n}-1$ disjoint subsets $X_{k}$ 's such that $\left|X_{k}\right|=R_{k}\left(B_{m}\right)$. For $X, Y \subseteq[N]$, denote $[Y, X]=\{Z \mid Y \subseteq Z \subseteq X\}$. Note that $[Y, X]$ is isomorphic to $\mathcal{B}_{|X \backslash Y|}$. Define $Y_{0}=\varnothing$. Since $\left[Y_{0}, X_{1}\right]$ is isomorphic to $\mathcal{B}_{m}$, there exists some set in $\left[Y_{0}, X_{1}\right]$ whose color is different from $c\left(Y_{0}\right)$. We pick such a set and call it $Y_{1}$. Similarly, we can find a set in $\left[Y_{0}, X_{2}\right]$ whose color is different from $c\left(Y_{0}\right)$ and $c\left(Y_{1}\right)$, otherwise the sets in $\left[Y_{0}, X_{2}\right]$ are colored by only two colors, which implies $\left[Y_{0}, X_{2}\right]$ contains a monochromatic 
$B_{m}$. We pick such a set and call it $Y_{2}$. Since $Y_{1} \subseteq X_{1}$ and $Y_{2} \subseteq X_{2}$, we have $Y_{1} \cap Y_{2}=$ $\varnothing$. Imitating the same operations, we can find pairwise disjoint $Y_{0}, \ldots, Y_{n}, Y_{i} \subset X_{i}$ for $1 \leqslant i \leqslant n$, of all distinct colors. These sets play the roles of $I_{0}, I_{1}, \ldots I_{n}$ of the rainbow $B_{n}$. For $n+1 \leqslant k \leqslant n+\left(\begin{array}{l}n \\ 2\end{array}\right)$, we have $\left|I_{k}\right|=2$. Suppose $I_{n+1}=\{i, j\}$. Then $\left[Y_{i} \cup Y_{j},\left(Y_{i} \cup Y_{j}\right) \cup X_{n+1}\right]$ is isomorphic to $\mathcal{B}_{R_{n+1}\left(B_{m}\right)}$. As before, we can find a set whose color is different from $c\left(Y_{0}\right), \ldots, c\left(Y_{n}\right)$ in $\left[Y_{i} \cup Y_{j},\left(Y_{i} \cup Y_{j}\right) \cup X_{n+1}\right]$, and choose it as the set $Y_{n+1}$. In general, we can find $Y_{k}$ in $\left[\cup_{i: I_{i} \subset I_{k}} Y_{i},\left(\cup_{i: I_{i} \subset I_{k}} Y_{i}\right) \cup X_{k}\right]$, whose color is different from $c\left(Y_{0}\right), c\left(Y_{1}\right), \ldots, c\left(Y_{k-1}\right)$, for $k \leqslant 2^{n}-1$. It is clear that if $I_{i} \subset I_{j}$, then $Y_{i} \subset Y_{j}$. Now if $I_{i}$ and $I_{j}$ are incomparable, then there exist some $i^{\prime} \in I_{i} \backslash I_{j}$ and $j^{\prime} \in I_{j} \backslash I_{i}$. Thus, $Y_{i^{\prime}} \subset\left(Y_{i} \backslash Y_{j}\right)$ and $Y_{j^{\prime}} \subset\left(Y_{j} \backslash Y_{i}\right)$, which implies that $Y_{i}$ and $Y_{j}$ are incomparable as well. As a conclusion, these sets $Y_{0}, \ldots, Y_{2^{n}-1}$ form a rainbow $B_{n}$ in $\mathcal{B}_{N}$.

Remark. By Theorem 3 (Theorem $5,[2]$ ), $R_{k}(P) \leqslant C \cdot k$, where $C$ is a constant determined by $P$ only. Thus, Theorem 14 is a proof of the existence of $R R(P, Q)$ without using Theorem 5. We will have more discussions on this aspect in next section.

Now we determine $R R\left(B_{m}, B_{n}\right)$ for $m=1$ or $n=1$.

Theorem 15. Let $m \geqslant 1$ and $n \geqslant 1$. For the Boolean posets, we have

$$
R R\left(B_{m}, B_{n}\right)=\left\{\begin{aligned}
m, & \text { if } n=1 \\
2^{n}-1, & \text { if } m=1 .
\end{aligned}\right.
$$

Proof. First, we show $R R\left(B_{m}, B_{1}\right)=m$ for $m \geqslant 1$. For $N<m$, we color every set in $\mathcal{B}_{N}$ with the same color to avoid a monochromatic $B_{m}$ and a rainbow $B_{1}$. On the other hand, the inequality in Theorem 14 shows that $R R\left(B_{m}, B_{1}\right) \leqslant R_{1}\left(B_{m}\right)=m$.

The next is $R R\left(B_{1}, B_{n}\right)=2^{n}-1$ for $n \geqslant 1$. For $N<2^{n}-1$, we can color all subsets in the level $\left(\begin{array}{c}{[N]} \\ i\end{array}\right)$ with $i$ for $1 \leqslant i \leqslant N$. Then every color class is an antichain. Moreover, to have a rainbow $B_{n}$, we need at least $2^{n}$ different colors, which is greater than the number of colors in the coloring. Therefore, no rainbow $B_{n}$ under this coloring, and we have $R R\left(B_{1}, B_{n}\right) \geqslant 2^{n}-1$.

By Theorem 14, $R R\left(B_{1}, B_{n}\right) \leqslant 2^{n-1}\left(2^{n}-1\right)$, which is far from the lower bound $2^{n}-1$ in the last paragraph. We will use an idea analogous to the proof of Theorem 14 to obtain the desired upper bound. For $N \geqslant 2^{n}-1$, let $S$ be the union of two disjoint sets $\left\{x_{1}, \ldots, x_{n}\right\}$ and $[N-n]$. Since $\mathcal{B}_{S}$ is isomorphic to $\mathcal{B}_{N}$, we can consider colorings of $\mathcal{B}_{S}$. Assume $\mathcal{B}_{S}$ does not contain a monochromatic $B_{1}$ under a given coloring $c$. Then we will prove that it contains a rainbow $B_{n}$ under $c$. Our strategy is to inspect the colors of the subsets of $\left\{x_{1}, \ldots, x_{n}\right\}$ one by one. If all the subsets of $\left\{x_{1}, \ldots, x_{n}\right\}$ are of different colors, then we are done. Once the color of a set $X \subset\left\{x_{1}, \ldots, x_{n}\right\}$ already appeared before, then we look for a new color in a chain which contains $X$ as the minimal element.

Let us arrange all subsets of $\left\{x_{1}, \ldots, x_{n}\right\}$ in the order $X_{0}, X_{1}, \ldots, X_{2^{n}-1}$ such that $\left|X_{k^{\prime}}\right| \leqslant\left|X_{k}\right|$ if $k^{\prime}<k$. First define $Y_{0}=X_{0}=\varnothing$ and $m_{0}=0$. For $k \geqslant 1$, let us define $m_{k} \leqslant N-n$ the smallest integer such that $m_{k} \geqslant m_{k^{\prime}}$ for any $X_{k^{\prime}} \subset X_{k}$ and $c\left(X_{k} \cup\left[m_{k}\right]\right)$ is different from any color in $\left\{c\left(Y_{0}\right), c\left(Y_{1}\right), \ldots, c\left(Y_{k-1}\right)\right\}$. Once such an integer exists, then 
define $Y_{k}=X_{k} \cup\left[m_{k}\right]$. By the definition of $Y_{k}$ and $m_{k}, Y_{k^{\prime}} \subset Y_{k}$ if and only if $X_{k^{\prime}} \subset X_{k}$. Hence the sets $Y_{0}, Y_{1} \ldots, Y_{2^{n}-1}$ form a rainbow $B_{n}$.

Claim: For $0 \leqslant k \leqslant 2^{n}-1, m_{k}$ exists.

Suppose, on the contrary, some $m_{k}$ does not exist. Let $k_{0}$ be the smallest $k$ such that $m_{k}$ does not exist. For any $k<k_{0}$, we call a chain $\mathcal{C}_{k}$ in $\mathcal{B}_{S}$ a principal chain of $Y_{k}$ if it consists of $\left|Y_{k}\right|+1$ sets and the largest one is $Y_{k}$ such that all the colors of the sets in $\mathcal{C}_{k}$ are in $\left\{c\left(Y_{0}\right), c\left(Y_{1}\right), \ldots, c\left(Y_{k}\right)\right\}$. We show the principal chain exists for all $Y_{k}$ with $k<k_{0}$. It is clear $\mathcal{C}_{0}=\{\varnothing\}$ is the principal chain of $Y_{0}$. For $Y_{k}$ with $k \geqslant 1$, let $k^{*}=\max \left\{k^{\prime} \mid X_{k^{\prime}} \subset X_{k}\right\}$. By induction, the principal chain $\mathcal{C}_{k^{*}}$ of $Y_{k^{*}}$ exists, and all the colors of the sets in $\mathcal{C}_{k^{*}}$ are in $\left\{c\left(Y_{0}\right), c\left(Y_{1}\right), \ldots, c\left(Y_{k^{*}}\right)\right\}$. Moreover, by the definition of $m_{k}$, the colors $c\left(X_{k} \cup[i]\right), m_{k^{*}} \leqslant i \leqslant m_{k}-1$, have appeared in $\left\{c\left(Y_{0}\right), c\left(Y_{0}\right), \ldots, c\left(Y_{k-1}\right)\right\}$. Note that $\mathcal{C}_{k^{*}}$ is the principal chain of $Y_{k^{*}}=X_{k^{*}} \cup\left[m_{k^{*}}\right]$ and we have $\left|X_{k^{*}}\right|=\left|X_{k}\right|-1$ by the choice of $k^{*}$. We can concatenate $\mathcal{C}_{k^{*}}$ to the chain $\left\{X_{k} \cup[i] \mid m_{k^{*}} \leqslant i \leqslant m_{k}\right\}$ to get a longer chain with

$$
\begin{aligned}
& \left|Y_{k^{*}}\right|+1+\left(m_{k}-m_{k^{*}}+1\right) \\
= & \left(\left|X_{k^{*}}\right|+m_{k^{*}}\right)+1+\left(m_{k}-m_{k^{*}}+1\right) \\
= & \left|X_{k}\right|+m_{k}+1 \\
= & \left|Y_{k}\right|+1
\end{aligned}
$$

sets. This is the principal chain of $Y_{k}$. Now since $m_{k_{0}}$ does not exist, it means the colors $c\left(X_{k_{0}} \cup[i]\right), m_{k^{* *}} \leqslant i \leqslant N-n$, are all in $\left\{c\left(Y_{0}\right), c\left(Y_{1}\right), \ldots, c\left(Y_{k_{0}-1}\right)\right\}$, where $k^{* *}=$ $\max \left\{k^{\prime} \mid X_{k^{\prime}} \subset X_{k_{0}}\right\}$. In other words, the number of the colors of the subsets in the chain $\mathcal{C}_{k^{* *}} \cup\left\{X_{k_{0}} \cup[i] \mid m_{k^{* *}} \leqslant i \leqslant N-n\right\}$ is at most $k_{0}$. Note that any chain under the coloring $c$ is a rainbow chain since we assume there is no monochromatic $B_{1}$. So we have $\left|Y_{k^{* *}}\right|+1+\left(N-n-m_{k^{* *}}+1\right) \leqslant k_{0}$. Since $\left|Y_{k^{* *}}\right|=\left|X_{k^{* *}}\right|+m_{k^{* *}}$, we have $N \leqslant n+k_{0}-\left|X_{k^{* *}}\right|-2$. By the choice of $k^{* *}$, we also have $\left|X_{k^{* *}}\right|=\left|X_{k_{0}}\right|-1$. In addition, $k_{0} \leqslant \sum_{i=1}^{\left|X_{k_{0}}\right|}\left(\begin{array}{l}n \\ i\end{array}\right)$ according to the ordering of the $X_{i}$ 's. Then

$$
N \leqslant n+\sum_{i=1}^{\left|X_{k_{0}}\right|}\left(\begin{array}{c}
n \\
i
\end{array}\right)-\left|X_{k_{0}}\right|-1<2^{n}-1 .
$$

This contradicts our assumption of $N$. As a conclusion, we have $R R\left(B_{1}, B_{n}\right) \leqslant 2^{n}-1$.

The idea of the principal chains in the proof of Theorem 15 can be applied to determine the Boolean rainbow Ramsey number for mixed types of posets below:

Theorem 16. For the chains and Boolean posets, we have $R R\left(C_{m}, B_{n}\right)=(m-1)\left(2^{n}-1\right)$.

Proof. For $N<(m-1)\left(2^{n}-1\right)$, we give a coloring of $\mathcal{B}_{N}$ by assigning color $i$ to the sets in the consecutive $m-1$ levels $\left(\begin{array}{c}{[N]} \\ (i-1)(m-1)\end{array}\right) \cup\left(\begin{array}{c}{[N]} \\ (i-1)(m-1)+1\end{array}\right) \cup \cdots \cup\left(\begin{array}{c}{[N]} \\ i(m-1)-1\end{array}\right)$ for $1 \leqslant i \leqslant\left\lceil\frac{N}{m-1}\right\rceil$. On the one hand, since a color class contains at most $m-1$ subsets of different sizes, $\mathcal{B}_{N}$ does not contain a monochromatic $C_{m}$. On the other hand, the number of colors is at most $2^{n}-1$. Hence it does not contain a rainbow $B_{n}$. 
Now for $N \geqslant(m-1)\left(2^{n}-1\right)$ we define $\mathcal{B}_{S}=\left\{x_{1}, x_{2}, \ldots, x_{n}\right\} \cup[N-n]$ as in the proof of Theorem 15, and study the colorings of $\mathcal{B}_{S}$. For a coloring $c$ of $\mathcal{B}_{S}$, we prove that once there is no monochromatic $C_{n}$, then there is a rainbow $B_{n}$ in $\mathcal{B}_{S}$ under $c$. Again, we arrange all subsets of $\left\{x_{1}, x_{2}, \ldots, x_{n}\right\}$ as $X_{0}, \ldots, X_{2^{n}-1}$ with $\left|X_{k^{\prime}}\right| \leqslant\left|X_{k}\right|$ if and only if $k^{\prime} \leqslant k$. Let $Y_{0}=X_{0}=\varnothing$ and $m_{0}=0$. Then for all $1 \leqslant k \leqslant 2^{n}-1$, define $Y_{k}=X_{k} \cup\left[m_{k}\right]$, where $m_{k} \leqslant N-n$ is the least integer such that $m_{k} \geqslant m_{k^{\prime}}$ whenever $X_{k^{\prime}} \subset X_{k}$ and $c\left(X_{k} \cup\left[m_{k}\right]\right)$ is a color different from $c\left(Y_{0}\right), \ldots, c\left(Y_{k-1}\right)$. Now it suffices to show all $Y_{k}$ 's exist as before. If some $Y_{k}$ does not exist, and $Y_{k^{\prime}}$ exists for all $k^{\prime}<k$, then we build up the principal chains $\mathcal{C}_{k^{\prime}}$ of $Y_{k^{\prime}}$ with the properties: $\left|\mathcal{C}_{k^{\prime}}\right|=\left|Y_{k^{\prime}}\right|+1$, the maximal set is $Y_{k^{\prime}}$, and the color of each set in $\mathcal{C}_{Y_{k^{\prime}}}$ is in $\left\{c\left(Y_{0}\right), c\left(Y_{1}\right), \ldots, c\left(Y_{k^{\prime}}\right)\right\}$. The only difference is now the principal chain is not necessarily rainbow. Nevertheless, the number of subsets of the same color in each $\mathcal{C}_{k^{\prime}}$ is at most $m-1$. Thus, if $Y_{k}$ does not exist, then the chain $\mathcal{C}_{k^{*}} \cup\left\{X_{k} \cup\left[m_{k^{*}}\right], X_{k} \cup\left[m_{k^{*}}+1\right], \ldots, X_{k} \cup[N-n]\right\}$ contains at most $(m-1) k$ subsets, where $m_{k^{*}}=\max \left\{m_{k^{\prime}} \mid X_{k^{\prime}} \subset X_{k}\right\}$. Hence, we have $\left|Y_{k^{*}}\right|+1+N-n-m_{k^{*}} \leqslant(m-1) k$, and

$$
\begin{aligned}
N & \leqslant(m-1) k-\left|Y_{k^{*}}\right|-1+n+m_{k^{*}} \\
& \leqslant(m-1) \sum_{i=1}^{\left|X_{k}\right|}\left(\begin{array}{c}
n \\
i
\end{array}\right)-\left[\left(\left|X_{k}\right|-1\right)+m_{k^{*}}+1\right]-1+n+m_{k^{*}} \\
& \leqslant(m-1) \sum_{i=1}^{\left|X_{k}\right|}\left(\begin{array}{c}
n \\
i
\end{array}\right)+n-\left|X_{k}\right|-1 \\
& <(m-1)\left(2^{n}-1\right) .
\end{aligned}
$$

This is again a contradiction. So $\mathcal{B}_{S}$ must contain a rainbow $B_{n}$ under $c$.

\section{Discussions}

We gave an upper bound for $R R\left(A_{m}, C_{n}\right)$ in Theorem 11. A lower bound can be deduced in the following way: Recall that $N_{m, n}$ is the minimum integer $N$ such that $\left(\begin{array}{c}N \\ \lfloor N / 2\rfloor\end{array}\right) \geqslant(m-$ $1)(n-1)+1$. Now consider the set $S$ that is a union of disjoint sets $X=\left\{x_{1}, \ldots, x_{N_{m, 2}-1}\right\}$ and $Y=\left\{y_{1}, \ldots, y_{n-2}\right\}$. For each subset $Z \subseteq S$, color it according to $Z \cap Y$. That is, we color $Z_{1}$ and $Z_{2}$ with the same color if and only if $Z_{1} \cap Y=Z_{2} \cap Y$. Thus, each color class is isomorphic to a Boolean poset $B_{N_{m, 2}-1}$ which does not contain $A_{m}$. On the other hand, if $Z_{1}, \ldots, Z_{k}$ form a rainbow chain, then $Z_{1} \cap Y, \ldots, Z_{k} \cap Y$ also form a rainbow chain. However, any rainbow chain formed by subsets of $Y$ has length at most $n-1$. So there does not exist a rainbow $C_{n}$. Therefore, we have $R R\left(A_{m}, C_{n}\right) \geqslant n-2+N_{m, 2}$. Combing the upper bound in Theorem 11, we have

$$
n-2+N_{m, 2} \leqslant R R\left(A_{m}, C_{n}\right) \leqslant(m-1)(n-2)+N_{m, 2} .
$$

Obviously, there is a huge gap between the lower and upper bounds. It would be interesting if one can tighten the gap. 
One of our main interests is to estimate $R R\left(B_{m}, B_{n}\right)$. Theorem 5 provides us an upper bound $R R\left(B_{m}, B_{n}\right) \leqslant n 2^{(2 n+1) 2^{m-1}-2}$. We also have $R R\left(B_{m}, B_{n}\right) \leqslant \sum_{i=1}^{2^{n}-1} R_{i}\left(B_{m}\right)$. In the following, we give an estimation of $\sum_{i=1}^{2^{n}-1} R_{i}\left(B_{m}\right)$ in terms of $m$ and $n$. Recall that in the Remark after Theorem 14, we mentioned that $R_{k}(P) \leqslant C \cdot k$, where $C$ is a constant determined by $P$. Indeed, this is a consequence from a result of Méroueh in [16]. Let us introduce the Lubell functions and forbidden subposet problems studied intensively recently. The Lubell function $\bar{h}_{n}(\mathcal{F})$ for a family $\mathcal{F}$ of subsets of $[n]$ is defined as

$$
\bar{h}_{n}(\mathcal{F}):=\sum_{F \in \mathcal{F}} \frac{1}{\left(\begin{array}{c}
n \\
|F|
\end{array}\right)} .
$$

It is obvious that $\bar{h}_{n}\left(\mathcal{B}_{n}\right)=n+1$. For the background of the Lubell functions and forbidden subposet problems, we refer the readers to a recent survey [11]. In the literature, Lu and Milans [14] proposed the conjecture:

Conjecture 17. [14] Let $\lambda_{n}(P)$ be the maximum value of the Lubell function for families of subsets of $[n]$ not containing $P$ as a subposet. Then $\lim \sup \lambda_{n}(P)$ is finite.

Méroueh [16] verified this conjecture by showing that if $\bar{h}_{n}(\mathcal{F})>1000 m^{7} 16^{m}$, then the family $\mathcal{F}$ must contain $B_{m}$, as well as any poset whose 2 -dimension is at most $m$. Thus, when $N \geqslant 1000 m^{7} 16^{m} k$, any $k$-coloring of $\mathcal{B}_{N}$ must contain one color class $\mathcal{F}_{i}$ of subsets of color $i$ with $\bar{h}\left(\mathcal{F}_{i}\right)>1000 m^{7} 16^{m}$, and hence a monochromatic $B_{m}$ of color $i$. In other words, $R_{k}\left(B_{m}\right) \leqslant 1000 m^{7} 16^{m} k$. Using his result, we conclude that

$$
R R\left(B_{m}, B_{n}\right) \leqslant \sum_{i=1}^{2^{n}-1} R_{i}\left(B_{m}\right) \leqslant 2^{n-1}\left(2^{n}-1\right) 1000 m^{7} 16^{m}<m^{7} 2^{2 n+4 m+9} .
$$

This upper bound is a little better than that derived from Theorem 5 under certain circumstances. It will be a challenging problem to determine or give a better estimation of $R R\left(B_{m}, B_{n}\right)$ for both $m \geqslant 2$ and $n \geqslant 2$.

\section{Acknowledgments}

The authors would like to thank the anonymous referee for many useful comments and suggestions to make the paper more legible and organized. The research is also partially supported by Taiwanese-Hungarian Mobility Program of the Hungarian Academy of Sciences and by Ministry of Science and Technology Project-based Personnel Exchange Program.

\section{References}

[1] M. Axenovich, A. Gyárfás, H. Liu, and D. Mubayi, Multicolor Ramsey numbers for triple systems. Discrete Mathematics, 322 (2014) 69-77. 
[2] M. Axenovich and S. Walzer, Boolean lattices: Ramsey properties and embeddings. Order, 34(2) (2017) 287-298.

[3] N. G. de Bruijn, C. van T. Ebbenhorst, and D. Kruyswijk, On the set of divisors of a number. Nieuw Arch. Wiskunde (2), 23 (1951) 191-193.

[4] F.-H. Chang, D. Gerbner, W.-T. Li, A. Methuku, D. T. Nagy, B. PATkós, And M. Vizer, Rainbow Ramsey problems for the Boolean lattice. arXiv: 1809.08629.

[5] D. Conlon, J. Fox And B. Sudakov, Hypergraph Ramsey numbers. Journal of the American Mathematical Society, 23 (1) (2010) 247-266.

[6] D. Conlon and J. Fox, B. Sudakov, Recent developments in graph Ramsey theory. Surveys in Combinatorics 2015, 49-118.

[7] C. Cox, D. Stolee, Ramsey numbers for partially-ordered sets. Order, 35(3) (2017) 557-579.

[8] R. P. Dilworth, A Decomposition Theorem for Partially Ordered Sets. Annals of Mathematics, 51(1) (1950) 161-166.

[9] S. Fujita, C. Magnant, and K. Ozeki, Rainbow generalizations of Ramsey theory-a dynamic survey. Theory and Application of Graphs, (2014).

[10] C. Greene and D. J. Kleitman, Strong versions of Sperner's theorem. Journal of Combinatorial Theory, Series A, 20 (1976), 80-88.

[11] J. R. Griggs And W.-T. Li, Progress on poset-free families of subsets, in: Recent Trends in Combinatorics, (2016), 317-338.

[12] T. Johnston, L. Lu, And K. G. Milans, Boolean algebras and Lubell functions, Journal of Combinatorial Theory, Series A, 136 (2015) 174-183.

[13] H. A. Kierstead and W. T. Trotter, A Ramsey theoretic problem for finite ordered sets. Discrete Mathematics, 63(2-3) (1987) 217-223.

[14] L. Lu And K. G. Milans, Set families with forbidden subposets. Journal of Combinatorial Theory, Series A, 136 (2015) 126-142.

[15] L. Lu And J. C. Thompson, Poset Ramsey Numbers for Boolean Lattices. arXiv: 1909.08680 (2019).

[16] A. MÉroueH, A LYM inequality for induced posets. Journal of Combinatorial Theory, Series A, 155 (2018) 398-417.

[17] W. T. Trotter, Embedding finite posets in cubes. Discrete Mathematics 12 (2) (1975) 165-172.

[18] W. T. Trotter, Ramsey theory and partially ordered sets. Contemporary Trends in Discrete Mathematics, RL Graham, et al., eds., DIMACS Series in Discrete Mathematics and Theoretical Computer Science, 49, (1999) 337-347. 